\title{
Urinary Tract Infection in Multiple Sclerosis: closing an audit loop by co-design and innovation
}

\section{$\underline{\text { Introduction }}$}

Multiple sclerosis (MS) is a neuroinflammatory disease that affects about 120,000 in the United Kingdom (UK) (and over 2 million world-wide) and is the most common cause of neurological disability in young adults in temperate climates. ${ }^{1}$ 50-80\% of patients with MS (PwMS) develop genito-urinary dysfunction that is commonly multi-factorial in aetiology. ${ }^{2,3,4}$ This urinary dysfunction can increase the risk of developing urinary tract infections (UTI) in PwMS. Estimates of urinary tract infection (UTI) incidence vary in the literature from 18-80\% (depending on the population studied and timing of data collection in relation to stage of MS) and is one of the most common reasons for hospital admission in PwMS. ${ }^{2,5,6}$ The presence of LUT (lower urinary tract) dysfunction such as detrusor over-activity, detrusor sphincter dyssynergia, incomplete bladder emptying with large post void residuals, use of urinary catheters, presence of vesicouterine reflux and increasing use of immunosuppressants may increase the risk of UTI in PwMS. Functional difficulties such as difficulty with toileting and personal hygiene may also increase the risk. ${ }^{7,8}$ The development of UTI in PWMS can result in urosepsis and its potential sequelae which include hospital admission, systemic sepsis and multi-organ failure. It can also lead to increased mortality risk, decreased quality of life and considerable cost to the health system. ${ }^{6}$

University College London Partners (UCLP) MS Service Group in the United Kingdom commissioned an independent agency, The Neurological Commissioning Support (NCS) to collate health episode statistics (HES) data on the impact of UTI in MS. ${ }^{9}$ These sources include University College Hospitals London NHS Foundation Trust, the Royal Free London NHS Trust, Bart's Healthcare NHS Trust and other hospitals and Clinical Commissioning Groups (CCG) across the regional National Health Service (NHS) community services. The NCS obtained data from publicly available health episode statistics covering a period from 2009/2010 to 2012/2013 (inclusive). Data are given as actual data across CCGs and providers, and normalised data (weighted per 100,000 population) across CCGs. PwMS often develop UTI in an unpredictable fashion and subsequently seek assistance from hospital institutions so for these reasons that we have focused on the data from non-elective admissions. This report highlights UTI as being one of the most common causes of nonelective hospital admission for PwMS and illustrates the high expenditure for NHS providers that is associated with this issue. ${ }^{9}$

The NCS collated data for organisations falling within the UCLP region over the period of $2012-2013 .{ }^{9}$ UTI was a common cause of hospital admission (in terms of all admissions) where the secondary diagnosis was MS (figure 1) and was the most common cause of non-elective hospital admission during this period (figure 2) 
Figure 1: Top 20 primary conditions (total admissions) where the secondary diagnosis is multiple sclerosis, $2012 / 13^{9}$

Figure 2: Top 20 primary conditions (non-elective admissions) where the secondary diagnosis is multiple sclerosis, $2012 / 13^{9}$

In patients where UTI was the primary diagnosis (all patients had MS as a secondary diagnosis), UTI was the highest in terms of total cost to the health system over the 2012/2013 period (Figure 3). ${ }^{9}$ The total cost across this one year period was $>£ 800,000 .^{9}$

Figure 3: Top 20 primary conditions (in terms of cost) where the secondary diagnosis is multiple sclerosis, $2012 / 13^{9}$ 
A recent repeat of the audit by HealthIQ Limited® covering a 1-year period from April 2016-March 2017 for the same region showed that for non-elective admissions, UTI remained the most common primary diagnosis in PwMS with the number slightly increasing when compared to 2012/2013 (figure 4). Furthermore, for nonelective admissions where UTI was the primary diagnosis (MS the secondary diagnosis), the overall cost had increased from just over $£ 800,000$ in 2012/2013 to $£ 930,982$ in 2016/2017 (figure 5). ${ }^{10}$ It should also be noted that aspiration pneumonia was the third most common cause of non-elective admission in PwMS in 2012/2013. Data collected from 2016/2017 showed it was the second most common cause of non-elective admission in PwMS. This emphasises the vital need for community speech and language therapy to address this significant and growing problem for PwMS. We plan to look at this in the future.

Figure 4: 2016/2017 Top 20 comorbidities for non-elective admissions where MS is the secondary diagnosis ${ }^{10}$

Figure 5: Tariff costs for $2016 / 2017$ for non-elective admissions where MS is secondary diagnosis ${ }^{10}$ 
Emerging data suggests that UTI in PwMS causes significant burden to the patient, their families and the NHS. Data compiled from a variety of NHS trusts and clinical commissioning groups across the UK show that UTI is one of the most common reasons for elective and non-elective admissions to hospital. In the UK non-elective care accounts for approximately $46 \%$ of the overall spend on hospital care for PwMS and $14 \%$ of unplanned admissions relate to UTIs. The UK MS Society estimates the cost of MS to the UK economy is $£ 3.3$ to $£ 4.2$ billion each year. ${ }^{11}$

\section{Methodology/Solution:}

Better strategies are needed to decrease the growing burden of UTI in PWMS to avoid the high human and financial costs of such admissions. To understand the impact of UTI and unplanned admissions on the patient and family, the MS Consultant Nurse organised multidisciplinary team training on The Kings Fund evidencebased co-design (EBCD) multi-faceted methodology. EBCD is a systematic approach that has been used successfully in many different International health care settings. ${ }^{12}$ Co-design is a collaborative approach between patients and staff which ensures that services are designed to meet the needs of patients and not those of an organisation. The methodology recognises that the patient and their families are the experts on the journey, step by step, from start to finish. This authentic lived experience is valued and their solutions for change are recognised as valid and essential.

The UCLP team captured several patients, families and staff interviews on film with their informed consent. This stage was followed up by a joint stakeholder event where stakeholders examined the audit findings and watched a compilation video before taking part in open discussion about recurring themes. Key messages that evolved included that care was often fragmented, systems were hard to navigate, clinicians did not share information and people with MS would like support to self-manage where possible.

During the co-design workshops patients and families described difficulties in accessing expert timely advice between appointments. Access to expert advice between visits to specialist teams, during out of hours or at weekends has also been highlighted as an area of concern in several independent audits carried out by MS charities and others over the years. ${ }^{13,14,15}$

In our EBCD work people living with MS and their families were asked to imagine what care would look like if they lived in a Brillant Borough ${ }^{\odot}$ where care was organsied to meet their needs.

People told us that in 'Brillant Borough ${ }^{\odot}$ ' they would would have access to expert advice day and night, shorter waiting times to assessment, faster assessment and treatment of urinary infections and health proferssionals would share information in a secure and timely manner (figure 6)

Figure 6 


\section{Outcome:}

The 'Brilliant Borough ${ }^{\odot}$ ' concept is being tested as part of the NHS Innovation Acceleration (NIA) fellowship programme which supports the creation and testing of a new model of care called NeuroResponse. NeuroResponse is an innovation that combines technology and humanity to improve the quality of life of people living with long term neurological conditions such as MS. The concept of combinatorial innovation emerged to describe different innovations working together rather than, for example, a single drug or technology. The concept also goes beyond multiple different technologies and instead, describes combinations of types of innovations; for example technology, workforce, new approaches to patient engagement, and digital channels for service delivery, all of which may be needed to achieve real improvements in value. ${ }^{16}$ NeuroResponse supports patient empowerment, co-ordinated action and dialogue between primary and secondary care providers, carers, social care professionals and voluntary sector staff as required. The model also combines a number of digital innovations including anticipatory electronic care plans, digital dipstick screening tools, near home detection and treatment of UTIs to provide a responsive ,accessible service that meets the needs of patients and their family (figure7) .

Figure 7 Key features of NeuroResponse

We worked closely with district nurses and general practitioners (GPs) to test NeuroResponse in a proof of concept study. Working with key partners we actively delivered the new care model to a pilot cohort of 5 people with complex MS needs - defined as having an expanded disability status scale (EDSS ) score of 8 or above and a history of urgent admissions in the London Borough of Camden. Three of the five had a history of unplanned admissions related to infections, with a total of 12 A\&E presentations with UTI which resulted in 12 unplanned admissions and a combined total of 65 bed days in an acute hospital setting from 2014-2016. None of them have had a further admission in the 17 months the pilot has been running.

\section{Conclusion and next steps:}

During the development and delivery of NeuroResponse to our cohort in Camden, we have gained a significant team of supporters, from patients and their families to the voluntary sector and health professionals within primary, community, acute, urgent and specialist care. A scale up to offer the service to 500 PwMS is now underway. 


\section{References:}

1. Mackenzie IS, Morant SV, Bloomfield GA, MacDonald TM, O'Riordan, J. Incidence and prevalence of multiple sclerosis in the UK 1990-2010: a descriptive study in the General Practice Research Database. Journal of neurology, neurosurgery, and psychiatry 85, 76-84 (2014).

2. de Seze M, Ruffion A, Denys $P$ et al. The neurogenic bladder in multiple sclerosis: review of the literature and proposal of management guidelines. Multiple Sclerosis Journal 13, 915-928 (2007).

3. Amarenco $\mathrm{G}$, Kerdraon J,Denys $\mathrm{P}$ et al Bladder and sphincter disorders in multiple sclerosis. Clinical, urodynamic and neurophysiological study of 225 cases. Revue neurologique 151, 722-30 (1995).

4. Akkoç $\mathrm{Y}$, Ersoz $\mathrm{M}$, Yuceyar $\mathrm{N}$ et al. Overactive bladder symptoms in patients with multiple sclerosis: Frequency, severity, diagnosis and treatment. The Journal of spinal cord medicine 39, 229-33 (2016).

5. Manack A, Motsko SP, Haag-Molkenteller $\mathrm{C}$ et al. Epidemiology and healthcare utilization of neurogenic bladder patients in a US claims database. Neurourology and urodynamics 30, 395-401 (2011).

6. Jick SS, Li L, Falcone, GJ et al. Epidemiology of multiple sclerosis: results from a large observational study in the UK. Journal of Neurology 262, 2033-2041 (2015).

7. Phé V, Pakzad M, Curtis $\mathrm{C}$ et al. Urinary tract infections in multiple sclerosis. Multiple sclerosis Journal 22: 855-861 (2016)

8. Vasudeva $\mathrm{P}$, Madersbacher $\mathrm{H}$. Factors implicated in pathogenesis of urinary tract infections in neurogenic bladders: some revered, few forgotten, others ignored. Neurourology and urodynamics 33, 95-100 (2014).

9. University College London Partners \& Neurological Commissioning Support. Discussion report Multiple sclerosis data. (2014).

10. Health IQ Limited. Health episode statistics in multiple sclerosis. (2018).

11. Losseff N, Oates M, Baines C, Keely E. London Strategic Clinical Network for Neurology CCG data reports. (2016)

12. The Point of Care Foundation. 1. What is Experience-based co-design? | Point of Care Foundation. Available at: https://www. pointofcarefoundation.org.uk/resource/experience-based-co-design-ebcdtoolkit/step-by-step-guide/1-experience-based-co-design/. (Accessed: 25th May 2018)

13. Diane Redfern-Tofts, L., McDougal, A. \& McDougal, W. My MS My Needs 2016: Access to treatment and health care (Technical report). (2016).

14. Royal College of Physicians. The national audit of services for people with multiple sclerosis. (2015).

15. National Audit Office. Services for people with neurological conditions. (2015)

16. NHS England. Real world testing of 'combinatorial innovation'. Available at https://www.england.nhs.uk/wp-content/uploads/2015/03/test-bed-prospectus.pdf. Accessed 11 June, 2018 